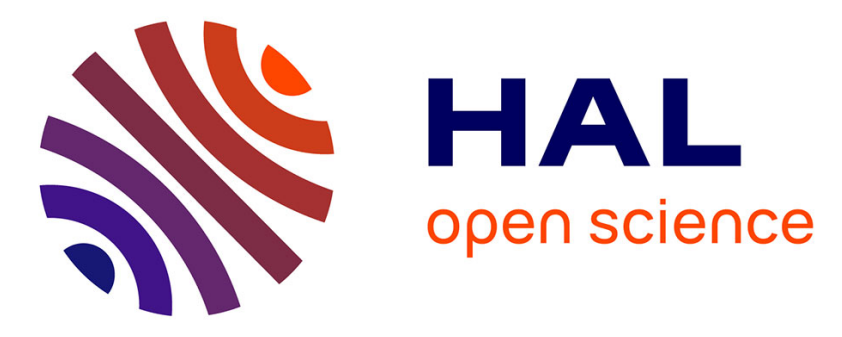

\title{
Long-range mediated interactions in a mixed-dimensional system
}

Daniel M Suchet, Zhigang Wu, Frédéric M Chevy, Georg M. Bruun

\section{To cite this version:}

Daniel M Suchet, Zhigang Wu, Frédéric M Chevy, Georg M. Bruun. Long-range mediated interactions in a mixed-dimensional system. Physical Review A : Atomic, molecular, and optical physics [19902015], 2017, 95 (4), pp.043643. 10.1103/PhysRevA.95.043643 . hal-01526933

\section{HAL Id: hal-01526933 https://hal.sorbonne-universite.fr/hal-01526933}

Submitted on 23 May 2017

HAL is a multi-disciplinary open access archive for the deposit and dissemination of scientific research documents, whether they are published or not. The documents may come from teaching and research institutions in France or abroad, or from public or private research centers.
L'archive ouverte pluridisciplinaire HAL, est destinée au dépôt et à la diffusion de documents scientifiques de niveau recherche, publiés ou non, émanant des établissements d'enseignement et de recherche français ou étrangers, des laboratoires publics ou privés. 


\title{
Long-range mediated interactions in a mixed-dimensional system
}

\author{
Daniel Suchet, ${ }^{1}$ Zhigang Wu, ${ }^{2, *}$ Frédéric Chevy, ${ }^{1}$ and Georg M. Bruun ${ }^{3}$ \\ ${ }^{1}$ Laboratoire Kastler Brossel, ENS-PSL Research University, CNRS, UPMC, Collège de France, 24 rue Lhomond, 75005 Paris, France \\ ${ }^{2}$ Institute for Advanced Study, Tsinghua University, Beijing 100084, China \\ ${ }^{3}$ Department of Physics and Astronomy, Aarhus University, DK-8000 Aarhus C, Denmark
}

(Received 26 February 2017; published 28 April 2017)

\begin{abstract}
We present a mixed-dimensional atomic gas system to unambiguously detect and systematically probe mediated interactions. In our scheme, fermionic atoms are confined in two parallel planes and interact via exchange of elementary excitations in a three-dimensional background gas. This interaction gives rise to a frequency shift of the out-of-phase dipole oscillations of the two clouds, which we calculate using a strong-coupling theory taking the two-body mixed-dimensional scattering into account exactly. The shift is shown to be easily measurable for strong interactions and can be used as a probe for mediated interactions.
\end{abstract}

DOI: 10.1103/PhysRevA.95.043643

\section{INTRODUCTION}

Mediated interactions were originally introduced to provide a quantum-mechanical explanation for the peculiar "action at a distance" interactions such as gravity and electromagnetism and they now constitute a major overarching paradigm in physics. In particle physics, exchange of gauge bosons is responsible for the propagation of fundamental interactions [1]. In condensed matter, the attraction between the electrons in BCS superconductors arises from the exchange of lattice phonons [2], and it is speculated that the mechanism behind high- $T_{c}$ superconductivity lies in the exchange of spin fluctuations [3]. The concept of mediated interactions is also important in classical physics, where fluctuations of classical fields are responsible for phenomena such as the finite-temperature Casimir effect in electrodynamics [4] and in biophysics [5].

Ultracold atoms have emerged as a versatile platform for the investigation of many-body physics, and a host of schemes have been proposed to explore mediated interactions using these systems. For instance, mediated interactions lead to the formation of a $p$-wave superfluid in spin-imbalanced fermionic systems [6-9]; they are responsible for the formation of a topological superfluid with a high critical temperature in $2 \mathrm{D}$ systems [10-12], and in 1D quantum liquids they are shown to result in Casimir-like forces between impurities [13]. In most cases, however, the mediated interaction is weak and in competition with direct interactions between atoms, making its experimental observation challenging.

In this paper, we apply the mixed-dimensional setup proposed in [14] and illustrated in Fig. 1 to study mediated interactions. Specifically we consider two parallel layers located at $z_{1}=0$ and $z_{2}=d$, which contain an equal number of spin-polarized noninteracting fermions (A species). The layers are immersed in a uniform 3D gas of interacting spin-1/2 fermions (B species), which can be tuned through the BEC-BCS crossover. The presence of the $3 \mathrm{D}$ gas induces a mediated interaction between the A particles: one A particle will perturb locally the surrounding B particles thereby inducing excitations in the 3D gas, which in turn affects the dynamics

\footnotetext{
*zwu@mail.tsinghua.edu.cn
}

of a second A particle. If A particles are harmonically trapped, this mediated coupling leads to a beating between oscillations in the two planes. Measuring the beating frequency between the $2 \mathrm{D}$ clouds therefore gives access to the strength of mediated interaction. This scheme is similar to Coulomb drag experiments in bilayered electronic systems [15] that was recently generalized to the case of dipolar gases [16].

To analyze the dynamics of this system, we develop a systematic many-body theory for the mediated interplane interaction that includes the low-energy mixed-dimensional A-B scattering exactly. We then derive an expression for the associated interaction energy between the two planes and calculate the frequency of the out-of-phase dipole oscillations of the $2 \mathrm{D}$ clouds in the $x y$ plane. In the weak A-B interaction limit, our results recover the perturbative expression for a mediated interaction proportional to the density-density response function of the 3D gas. In the strong A-B interaction limit, however, the weak-coupling result breaks down completely. In the latter case we focus on the BEC regime of the 3D gas and show that the mediated interaction gives rise to a significant and easily detectable shift in the out-of-phase dipole oscillation frequency of the two clouds.

The rest of the paper is organized as follows. In Sec. II, we derive expressions for the interlayer mediated interaction for both weak and strong 2D-3D interaction strengths. In the latter case we focus on the BEC regime for the 3D gas. To do so, we discuss in detail the determination of the mixeddimensional scattering matrix. In Sec. III, the correction to the thermodynamic potential due to the mediated interaction is obtained. Furthermore, we use the local density approximation to generalize our result to the trapped 2D layers. In Sec. IV, a scheme to probe the mediated interaction is proposed by means of dipole oscillations of the 2D layers. The shift of the dipole oscillation frequency due to the presence of the mediated interaction is calculated using realistic experimental parameters and the possible outcomes of such a probe are discussed. Our findings are summarized in Sec. V.

\section{MEDIATED INTERACTION}

The interaction between the $\mathrm{A}$ and $\mathrm{B}$ particles is short range and can be characterized by an effective 2D-3D scattering length $a_{\text {eff }}$ [17]. In terms of the well-known $T$-matrix ap- 


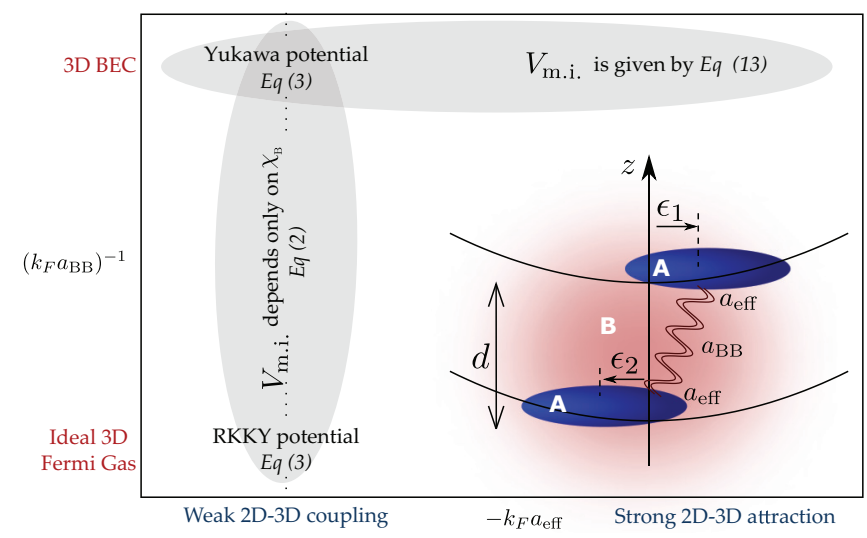

FIG. 1. We consider A fermions confined in two layers by two identical harmonic traps with a frequency $\omega_{z}$ much larger than any other energy scale in the system, and trapped in the $x y$ plane by a weak harmonic potential with frequency $\omega_{\perp}$. The two layers are immersed in a $3 \mathrm{D}$ cloud of spin- $1 / 2$ fermions (B atoms), which mediates an interaction between the two layers. This mediated interaction gives rise to a frequency shift of the out-of-phase dipole oscillation of the two A clouds, which depends on the B-B scattering length $a_{B B}$ as well as on the 2D-3D A-B scattering length $a_{\text {eff }}$. The ranges of $a_{\text {eff }}$ and $a_{B B}$ analyzed in this paper are indicated by the gray regions. The main focus of our paper is for a strong 2D-3D interaction and on the BEC side of the 3D gas with a dimer scattering length $a_{B}$.

proximation, the scattering amplitude $\mathcal{T}_{A B}$ satisfies an integral equation represented diagrammatically in Fig. 2. It can be shown that $\mathcal{T}_{A B}$ only depends on the total momentum and frequency of incoming particles $\mathbf{p}_{\perp}=\mathbf{p}_{1 \perp}+\mathbf{p}_{2 \perp}=\mathbf{p}_{3 \perp}+\mathbf{p}_{4 \perp}$ and $\omega_{v}=\omega_{n_{1}}+\omega_{\nu_{2}}=\omega_{n_{3}}+\omega_{\nu_{4}}$. Solving the integral equation yields

$$
\mathcal{T}_{A B}\left(\mathbf{p}_{\perp}, i \omega_{\nu}\right)=\frac{g}{1-g \Pi\left(\mathbf{p}_{\perp}, i \omega_{\nu}\right)},
$$

where $g=2 \pi a_{\text {eff }} / \sqrt{m_{B} m_{r}}$, and $m_{r}=m_{A} m_{B} /\left(m_{A}+m_{B}\right)$ is the reduced mass $\left(\hbar=k_{B}=1\right)$. Here $m_{A}$ denotes the mass of an $\mathrm{A}$ fermion and $m_{B}$ that of the scattering particle in the $3 \mathrm{D}$ gas, namely, the mass of the B fermion (dimer) in the BCS (BEC) regime. $\Pi\left(\mathbf{p}_{\perp}, i \omega_{\nu}\right)$ is the renormalized $2 \mathrm{D}-3 \mathrm{D}$ pair propagator for the center-of-mass $(\mathrm{COM})$ momentum $\mathbf{p}_{\perp}=$ $\left(p_{x}, p_{y}\right)$ in the plane, and $i \omega_{\nu}$ is either a bosonic (BCS regime) or fermionic (BEC regime) Matsubara frequency. Equation (1) includes many-body effects in the ladder approximation, and recovers the correct low-energy $2 \mathrm{D}-3 \mathrm{D}$ scattering matrix in a vacuum [18].

\section{A. Weak 2D-3D interaction}

In order to get a simple physical picture, we first consider the case of a weak 2D-3D interaction where $a_{\text {eff }}$ is much smaller than the interparticle spacing of the $\mathrm{A}$ and $\mathrm{B}$ particles. It then follows from Eq. (1) that $T_{A B}\left(\mathbf{p}_{\perp}, i \omega_{\nu}\right) \simeq g$, and second-order
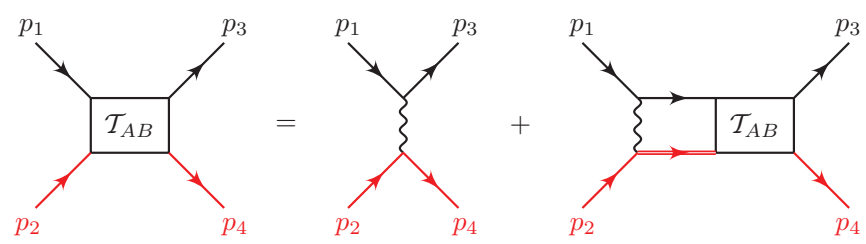

FIG. 2. Scattering $T$ matrix between a $2 \mathrm{D}$ fermion and a $3 \mathrm{D}$ particle. The black line represents the $2 \mathrm{D}$ fermion propagator and the red line represents the $3 \mathrm{D}$ particle propagator. Here $p_{1} \equiv$ $\left(\mathbf{p}_{1 \perp}, i \omega_{n_{1}}\right), p_{2} \equiv\left(\mathbf{p}_{2}, i \omega_{v_{2}}\right), p_{3} \equiv\left(\mathbf{p}_{3 \perp}, i \omega_{n_{3}}\right)$, and $p_{4} \equiv\left(\mathbf{p}_{4}, i \omega_{v_{4}}\right)$.

perturbation theory gives

$$
V_{\mathrm{mi}}\left(\mathbf{q}_{\perp}, i \omega_{\nu}\right)=g^{2} \int_{-\infty}^{\infty} d q_{z} e^{i q_{z} d} \chi_{B}\left(\mathbf{q}_{\perp}, q_{z}, i \omega_{\nu}\right)
$$

which describes the mediated interaction between two $\mathrm{A}$ particles in different planes. Here $\left(\mathbf{q}_{\perp}, i \omega_{\nu}\right)=\left(q_{x}, q_{y}, i \omega_{\nu}\right)$ are the transferred momentum and frequency and $\chi_{B}\left(\mathbf{q}_{\perp}, q_{z}, i \omega_{\nu}\right)$ is the density-density response function of the $\mathrm{B}$ cloud. The integration over the momentum $q_{z}$ comes from the fact that it is not conserved in the 2D-3D scattering. Deep in the BCS limit where the $\mathrm{B}$ fermions form an ideal Fermi gas, the mediated interaction Eq. (2) is of the form of a Ruderman-Kittel-KasuyaYosida potential $[14,19-21]$. When the B fermions are deep in the BEC limit where they form a weakly interacting BEC of dimers, the mediated interaction takes the form of a Yukawa potential [22]. At zero frequency, Fourier transforming (2) back to the real space gives

$$
V_{\mathrm{mi}}(r)= \begin{cases}g^{2} \frac{m_{B}}{16 \pi^{3}} \frac{2 p_{F} r \cos 2 p_{F} r-\sin 2 p_{F} r}{r^{4}}, & \text { BCS limit, } \\ -g^{2} \frac{n_{B} m_{B}}{\pi r} e^{-\sqrt{2} r / \xi_{B}}, & \text { BEC limit, }\end{cases}
$$

where $p_{F}$ is the Fermi momentum of the 3D Fermi gas in the BCS regime, and $n_{B}$ is the density of the $3 \mathrm{D}$ BEC of dimers with coherence length $\xi_{B}=1 / \sqrt{8 \pi n_{B} a_{B}}$. Here, $a_{B}=0.6 a_{B B}$ is the scattering length between the deeply bound dimers of $B$ fermions [23].

\section{B. Strong 2D-3D interaction}

For a strong 2D-3D interaction where $a_{\text {eff }}$ is comparable to or larger than the interparticle spacing, the mediated interaction between the two layers takes on a more complex form. The reason is that we need to retain the full COM momentum and frequency dependence of the $2 \mathrm{D}-3 \mathrm{D}$ scattering matrix $\mathcal{T}_{A B}$ given by Eq. (1).

We shall from now on concentrate on the BEC limit of the $\mathrm{B}$ fermions, namely when they form a weakly interacting BEC of dimers, which can be treated within Bogoliubov theory. In principle, the density of the $\mathrm{BEC}$ in the vicinity of the $2 \mathrm{D}$ layers will be affected by strong 2D-3D interaction. However, as we will see later, the mediated interaction is determined primarily by the bulk properties of the BEC. Thus we shall neglect any modification of the 3D BEC density due to the 2D-3D interaction in our following treatment. In this case, the renormalized pair propagator $\Pi\left(\mathbf{p}_{\perp}, i \omega_{\nu}\right)$ is given by

$$
\Pi\left(\mathbf{p}_{\perp}, i \omega_{\nu}\right)=\int \frac{d^{3} p^{\prime}}{(2 \pi)^{3}}\left[\frac{1}{\beta} \sum_{n} G_{j}^{\mathrm{A}}\left(\mathbf{p}_{-}, i \frac{m_{A}}{M} \omega_{\nu}-i \omega_{n}\right) G_{0}^{\mathrm{B}}\left(\mathbf{p}_{+}, i \frac{m_{A}}{M} \omega_{\nu}+i \omega_{n}\right)+\frac{1}{p_{z}^{\prime 2} / 2 m_{B}+\mathbf{p}_{\perp}^{\prime 2} / 2 m_{r}+i 0^{+}}\right]
$$


where $\mathbf{p}^{\prime}=\left(\mathbf{p}_{\perp}^{\prime}, p_{z}^{\prime}\right), \mathbf{p}_{+} \equiv \frac{m_{B}}{M} \mathbf{p}_{\perp}+\mathbf{p}^{\prime}, \mathbf{p}_{-} \equiv \frac{m_{A}}{M} \mathbf{p}_{\perp}-\mathbf{p}_{\perp}^{\prime}$. Here $G_{j}^{\mathrm{A}}\left(\mathbf{p}_{\perp}, i \omega_{m}\right)=1 /\left(i \omega_{m}-p_{\perp}^{2} / 2 m_{A}+\mu_{A}\right)$ is the Green's function for the A fermions in the $j$ th layer with $\mu_{A}$ being the chemical potential and $G_{0}^{\mathrm{B}}\left(\mathbf{q}, i \omega_{\nu}\right)=1 /\left(i \omega_{\nu}-p^{2} / 2 m_{B}\right)$ is the noninteracting boson Green's function. For weakly interacting bosons, it is a good approximation to use the noninteracting boson Green's function in place of the the normal boson Green's function in determining the pair propagator. We find at $T=0$

$$
\Pi\left(\mathbf{p}_{\perp}, i \omega_{\nu}\right)=\int \frac{d^{3} p^{\prime}}{(2 \pi)^{3}}\left[\frac{1-\Theta\left(k_{F}-\left|m_{A} \mathbf{p}_{\perp} / M-\mathbf{p}_{\perp}^{\prime}\right|\right)}{i \omega_{\nu}-\left(\mathbf{p}_{\perp}^{2} / 2 M+\mathbf{p}_{\perp}^{\prime 2} / 2 m_{r}+p_{z}^{\prime 2} / 2 m_{B}\right)+\mu_{A}}+\frac{1}{p_{z}^{\prime 2} / 2 m_{B}+\mathbf{p}_{\perp}^{\prime 2} / 2 m_{r}+i 0^{+}}\right],
$$

where $k_{F}=\sqrt{2 m_{A} \mu_{A}}$ is the Fermi momentum of the A species and $\Theta(x)$ is the Heaviside step function. Expressed in terms of the dimensionless variables, the pair propagator is

$$
\Pi\left(\mathbf{p}_{\perp}, i \omega_{\nu}\right)=2 m_{A} k_{F} \int \frac{d^{2} p_{\perp}^{\prime}}{(2 \pi)^{2}} \int \frac{d p_{z}^{\prime}}{2 \pi}\left[\frac{1-\Theta\left(1-\left|\mathbf{p}_{\perp}^{\prime}-\alpha_{A} \mathbf{p}_{\perp}\right|\right)}{i \omega_{\nu}-\left(\alpha_{A} p_{\perp}^{2}+\alpha_{B}^{-1} p_{\perp}^{\prime 2}+\alpha_{A} \alpha_{B}^{-1} p_{z}^{\prime 2}\right)+1}+\frac{1}{\alpha_{A} \alpha_{B}^{-1} p_{z}^{\prime 2}+\alpha_{B}^{-1} p_{\perp}^{\prime 2}+i 0^{+}}\right],
$$

where $\alpha_{A}=m_{A} / M$ and $\alpha_{B}=m_{B} / M$. Here the frequency variables are scaled in terms of the chemical potential $\mu_{A}$ and the momentum variables in terms of the Fermi momentum $k_{F}$. We write

$$
\Pi\left(\mathbf{p}_{\perp}, i \omega_{\nu}\right)=\Pi_{0}\left(\mathbf{p}_{\perp}, i \omega_{\nu}\right)+\Delta \Pi\left(\mathbf{p}_{\perp}, i \omega_{\nu}\right)
$$

where

$$
\begin{aligned}
\Pi_{0}\left(\mathbf{p}_{\perp}, i \omega_{\nu}\right) & \equiv 2 m_{A} k_{F} \int \frac{d^{2} p_{\perp}^{\prime}}{(2 \pi)^{2}} \int \frac{d p_{z}^{\prime}}{2 \pi}\left[\frac{1}{i \omega_{\nu}-\left(\alpha_{A} p_{\perp}^{2}+\alpha_{B}^{-1} p_{\perp}^{\prime 2}+\alpha_{A} \alpha_{B}^{-1} p_{z}^{\prime 2}\right)+1}+\frac{1}{\alpha_{A} \alpha_{B}^{-1} p_{z}^{\prime 2}+\alpha_{B}^{-1} p_{\perp}^{\prime 2}+i 0^{+}}\right] \\
& =-i \frac{m_{A} k_{F}}{2 \pi \alpha_{A}^{1 / 2} \alpha_{B}^{-3 / 2}} \sqrt{i \omega_{\nu}+1-\alpha_{A} p_{\perp}^{2}}
\end{aligned}
$$

is the pair propagator in vacuum and

$$
\begin{aligned}
\Delta \Pi\left(\mathbf{p}_{\perp}, i \omega_{\nu}\right) & \equiv-2 m_{A} k_{F} \int \frac{d^{2} p_{\perp}^{\prime}}{(2 \pi)^{2}} \int \frac{d p_{z}^{\prime}}{2 \pi} \frac{\Theta\left(1-\left|\mathbf{p}_{\perp}^{\prime}-\alpha_{A} \mathbf{p}_{\perp}\right|\right)}{i \omega_{\nu}-\left(\alpha_{A} p_{\perp}^{2}+\alpha_{B}^{-1} p_{\perp}^{\prime 2}+\alpha_{A} \alpha_{B}^{-1} p_{z}^{\prime 2}\right)+1} \\
& =i \frac{m_{A} k_{F}}{\sqrt{\alpha_{A} \alpha_{B}^{-1}}} \int \frac{d^{2} p_{\perp}^{\prime}}{(2 \pi)^{2}} \frac{\Theta\left(1-\left|\mathbf{p}_{\perp}^{\prime}-\alpha_{A} \mathbf{p}_{\perp}\right|\right)}{\sqrt{i \omega_{\nu}+1-\alpha_{A} p_{\perp}^{2}-\alpha_{B}^{-1} p_{\perp}^{\prime 2}}}
\end{aligned}
$$

is the medium correction due to the presence of the Fermi sea. Here $\sqrt{z}$ always denotes the root of the complex number $z$ that lies in the upper half plane.

From Eqs. (8) and (9) we find

$$
\Pi\left(\mathbf{p}_{\perp}, i \omega_{\nu}\right)=-i \frac{m_{A} k_{F}}{2 \pi^{2} \alpha_{A}{ }^{1 / 2} \alpha_{B}^{-3 / 2}} \int_{0}^{\pi / 2} d \theta\left(\sqrt{i \omega_{\nu}-\gamma_{+}\left(\theta, p_{\perp}\right)}-\sqrt{i \omega_{\nu}-\gamma_{-}\left(\theta, p_{\perp}\right)}\right)
$$

for $\alpha_{A} p_{\perp} \leqslant 1$. Here and in the following

$$
\gamma_{ \pm}\left(\theta, p_{\perp}\right) \equiv \alpha_{B}^{-1} p_{ \pm}^{2}(\theta)+\alpha_{A} p_{\perp}^{2}-1
$$

where $p_{ \pm}(\theta)= \pm \alpha_{A} p_{\perp} \cos \theta+\sqrt{1-\alpha_{A}^{2} p_{\perp}^{2} \sin ^{2} \theta}$. For $\alpha_{A} p_{\perp}>1$ we find

$$
\Pi\left(\mathbf{p}_{\perp}, i \omega_{\nu}\right)=-i \frac{m_{A} k_{F}}{2 \pi^{2} \alpha_{A}{ }^{1 / 2} \alpha_{B}^{-3 / 2}}\left[\sqrt{i \omega_{\nu}-\left(\alpha_{A} p_{\perp}^{2}-1\right)}+\frac{1}{\pi} \int_{0}^{\theta_{0}} d \theta\left(\sqrt{i \omega_{\nu}-\gamma_{+}\left(\theta, p_{\perp}\right)}-\sqrt{i \omega_{\nu}-\gamma_{-}\left(\theta, p_{\perp}\right)}\right)\right],
$$

where $\theta_{0}=\sin ^{-1}\left(1 / \alpha_{A} p_{\perp}\right)$. Equations (10) and (12) can be used in Eq. (1) to determine the scattering amplitude between the $2 \mathrm{D}$ fermion and the $3 \mathrm{D}$ boson for arbitrary 2D-3D interaction strength.

With this expression of $\mathcal{T}_{A B}$, we proceed to the derivation of the mediated interaction $V_{\mathrm{mi}}$. The mediated interaction between the A particles is calculated including all processes where a single Bogoliubov phonon in the BEC is exchanged between the two layers. In a diagrammatic language, these processes are shown in Fig. 3(a).

Summing up the contributions from the four terms in Fig. 3(a) gives

$$
\begin{aligned}
V_{\mathrm{mi}}\left(p_{1}, p_{2} ; q\right)= & n_{B} \mathcal{T}_{A B}\left(p_{1}+q\right) \mathcal{T}_{A B}\left(p_{2}\right) \bar{G}_{11}^{\mathrm{B}}\left(\mathbf{q}_{\perp}, i \omega_{\nu}\right)+n_{B} \mathcal{T}_{A B}\left(p_{1}\right) \mathcal{T}_{A B}\left(p_{2}-q\right) \bar{G}_{11}^{\mathrm{B}}\left(-\mathbf{q}_{\perp},-i \omega_{\nu}\right) \\
& +n_{B} \mathcal{T}_{A B}\left(p_{1}+q\right) \mathcal{T}_{A B}\left(p_{2}-q\right) \bar{G}_{12}^{\mathrm{B}}\left(\mathbf{q}_{\perp}, i \omega_{\nu}\right)+n_{B} \mathcal{T}_{A B}\left(p_{1}\right) \mathcal{T}_{A B}\left(p_{2}\right) \bar{G}_{21}^{\mathrm{B}}\left(\mathbf{q}_{\perp}, i \omega_{\nu}\right),
\end{aligned}
$$



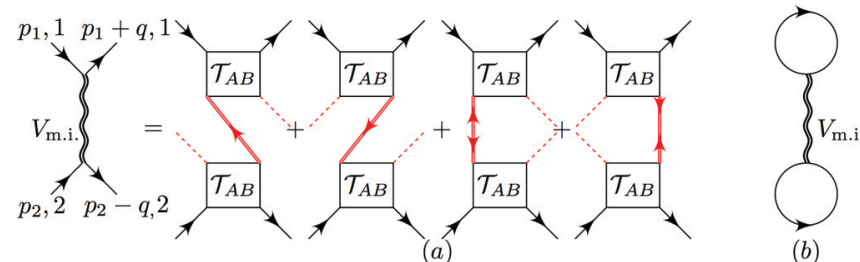

(b)

FIG. 3. (a) The mediated interaction $V_{\mathrm{mi}}$ between fermions in layers 1 and 2 coming from the exchange of one Bogoliubov mode. The box represents the $2 \mathrm{D}-3 \mathrm{D}$ scattering amplitude $\mathcal{T}_{A B}$, the dashed red line represents bosons emitted or absorbed by the condensate, and the red thick line with one arrow and two arrows represents the normal $G_{11}^{\mathrm{B}}$ and anomalous Green's functions $G_{12}^{\mathrm{B}}$ (or $G_{21}^{\mathrm{B}}$ ), respectively. (b) The leading correction to the thermodynamic potential due to the mediated interaction between the two planes. The thin solid lines represent the Fermi propagators in the two planes.

where $p_{1} \equiv\left(\mathbf{p}_{1 \perp}, i \omega_{m_{1}}\right), p_{2} \equiv\left(\mathbf{p}_{2 \perp}, i \omega_{m_{2}}\right)$, and $q \equiv\left(\mathbf{q}_{\perp}, i \omega_{\nu}\right)$. Here $\omega_{m}=(2 m+1) \pi / \beta$ and $\omega_{\nu}=2 \nu \pi / \beta$ are Fermi and Bose Matsubara frequencies, respectively, where $\beta=1 / T$ is the inverse temperature and $m$ and $v$ are integers. In Eq. (13), the Green's functions of the BEC are integrated over the $z$ component of the momentum as

$$
\bar{G}_{\alpha \beta}^{\mathrm{B}}\left(\mathbf{q}_{\perp}, i \omega_{\nu}\right) \equiv \int_{-\infty}^{\infty} \frac{d q_{z}}{2 \pi} G_{\alpha \beta}^{\mathrm{B}}\left(\mathbf{q}_{\perp}, q_{z}, i \omega_{\nu}\right) e^{i q_{z} d} .
$$

The Green's functions of the 3D BEC are as usual

$$
G_{11}^{\mathrm{B}}=\frac{u_{\mathbf{k}}^{2}}{i \omega_{v}-E_{\mathbf{k}}}-\frac{v_{\mathbf{k}}^{2}}{i \omega_{v}+E_{\mathbf{k}}}, \quad G_{12}^{\mathrm{B}}=\frac{g_{B} n_{B}}{\omega_{v}^{2}+E_{\mathbf{k}}^{2}},
$$

where $\quad \mathbf{k}=\left(\mathbf{k}_{\perp}, k_{z}\right) \quad$ and $G_{21}^{\mathrm{B}}\left(\mathbf{k}, i \omega_{\nu}\right)=G_{12}^{\mathrm{B}}\left(\mathbf{k}, i \omega_{\nu}\right)$. We have defined $u_{\mathbf{k}}^{2}, v_{\mathbf{k}}^{2}=\frac{1}{2}\left[\left(\epsilon_{\mathbf{k}}+g_{B} n_{B}\right) / E_{\mathbf{k}} \pm 1\right], E_{\mathbf{k}}=$ $\sqrt{\epsilon_{\mathbf{k}}\left(\epsilon_{\mathbf{k}}+2 g_{B} n_{B}\right)}$ is the Bogoliubov spectrum with $\epsilon_{\mathbf{k}}=k^{2} / 2 m_{B}$, and $g_{B}=4 \pi a_{B} / m_{B}$. Note that the mediated interaction Eq. (13) depends on both $p_{1}$ and $p_{2}$ as well as $q$ due to the momentum and frequency dependence of the 2D-3D scattering. In fact, in the weak-interaction limit $\mathcal{T}_{A B} \simeq g$, one recovers Eq. (2) from the more general expression Eq. (13).

\section{THERMODYNAMICAL POTENTIAL}

\section{A. Uniform 2D layers}

We now derive an expression for the correction to the thermodynamic potential $\Omega$ due to the mediated interaction between the two planes for a general strength of the 2D-3D interaction. The dominant contribution is the Hartree term illustrated in Fig. 3(b). For a homogeneous system, this term gives the correction per unit area as (for the rest of the paper the $\perp$ subscript will be dropped in the vector notation and all boldface letters now denote in-plane $2 \mathrm{D}$ vectors)

$$
\begin{aligned}
\bar{\Omega}_{\mathrm{mi}}= & \frac{1}{\beta^{2}} \sum_{m_{1} m_{2}} \int \frac{d^{2} p_{1}}{(2 \pi)^{2}} \frac{d^{2} p_{2}}{(2 \pi)^{2}} V_{\mathrm{mi}}\left(p_{1}, p_{2} ; 0\right) \\
& \times G_{1}^{\mathrm{A}}\left(\mathbf{p}_{1}, i \omega_{m_{1}}\right) G_{2}^{\mathrm{A}}\left(\mathbf{p}_{2}, i \omega_{m_{2}}\right),
\end{aligned}
$$

where $G_{j}^{\mathrm{A}}\left(\mathbf{p}, i \omega_{m}\right)=1 /\left(i \omega_{m}-p^{2} / 2 m_{A}+\mu_{A}\right)$ is the Green's function for the $\mathrm{A}$ fermions in the $j$ th layer. Using Eq. (13) together with the identity $2 \bar{G}_{11}(0,0)+2 \bar{G}_{12}(0,0)=$ $-\sqrt{2} n_{B} m_{B} \xi_{B} \exp \left(-\sqrt{2} d / \xi_{B}\right)$ yields

$$
\bar{\Omega}_{\mathrm{mi}}=-\sqrt{2} m_{B} \xi_{B} n_{B} e^{-\sqrt{2} d / \xi_{B}} \bar{\Omega}_{1} \bar{\Omega}_{2},
$$

where

$$
\bar{\Omega}_{j}=\frac{1}{\beta} \sum_{m} \int \frac{d^{2} p}{(2 \pi)^{2}} \mathcal{T}_{A B}\left(\mathbf{p}, i \omega_{m}\right) G_{j}^{\mathrm{A}}\left(\mathbf{p}, i \omega_{m}\right) .
$$

The Matsubara frequency summation in the above expression can in fact be performed analytically, which greatly simplifies the numerical calculation of thermodynamic potential density. Substituting Eq. (1) in Eq. (18) and using the dimensionless momenta and frequencies introduced earlier, we obtain

$$
\begin{aligned}
\bar{\Omega}_{j} & =2 \frac{g m_{A}}{\beta} \sum_{m} \int \frac{d^{2} p}{(2 \pi)^{2}} \frac{1}{\left[1-g \Pi\left(\mathbf{p}, i \omega_{m}\right)\right]\left[i \omega_{m}-\left(p^{2}-1\right)\right]} \\
& =\frac{g m_{A}}{\pi \beta} \sum_{m} \int_{0}^{\infty} d p \frac{1}{\left[1-g \Pi\left(\mathbf{p}, i \omega_{m}\right)\right]\left[i \omega_{m}-\left(p^{2}-1\right)\right]} .
\end{aligned}
$$

Inserting Eqs. (10) and (12) into Eq. (19), and performing the Matsubara frequency summation, we find for negative 2D-3D scattering length $a_{\text {eff }}<0$ and in the zero-temperature limit $\beta \rightarrow \infty$

$$
\bar{\Omega}_{j}=2 a_{\mathrm{eff}} \alpha_{A}^{1 / 2} \alpha_{B}^{-1} \mu_{A} \int_{0}^{1} d p S(p)
$$

where

$$
S(p)=\frac{1}{1-k_{F} a_{\mathrm{eff}} \sqrt{\alpha_{B}} \frac{1}{\pi} \int_{0}^{\pi / 2} d \theta\left[\sqrt{1-p^{2}+\gamma_{+}(\theta, p)}+\sqrt{1-p^{2}+\gamma_{-}(\theta, p)}\right]} .
$$

\section{B. Trapped 2D layers: Local-density approximation}

Using the local-density approximation, we can generalize Eq. (16), which was derived assuming a homogeneous system, to the case of trapped 2D Fermi clouds. This yields

$$
\Omega_{\mathrm{mi}}\left(\epsilon_{1}-\epsilon_{2}\right)=\int d^{2} r_{1} d^{2} r_{2}\left[2 \bar{G}_{11}^{\mathrm{B}}\left(\mathbf{r}_{1}-\mathbf{r}_{2}, 0\right)+2 \bar{G}_{12}^{\mathrm{B}}\left(\mathbf{r}_{1}-\mathbf{r}_{2}, 0\right)\right] \bar{\Omega}_{1}\left(\mathbf{r}_{1}-\epsilon_{1} \hat{\mathbf{x}}\right) \bar{\Omega}_{2}\left(\mathbf{r}_{2}-\epsilon_{2} \hat{\mathbf{x}}\right),
$$

where $\bar{G}_{i j}^{\mathrm{B}}(\mathbf{r}, 0)$ is the $2 \mathrm{D}$ Fourier transform of $\bar{G}_{i j}^{\mathrm{B}}(\mathbf{p}, 0)$ back to real $2 \mathrm{D}$ space, and $\bar{\Omega}_{i}(\mathbf{r})$ is given by Eq. (18) using a local chemical potential $\mu_{A}(\mathbf{r})=\mu_{A}+m_{A} \omega_{\perp}^{2} r^{2} / 2$. In Eq. (22), we have allowed the two A clouds to be rigidly displaced distances of 
$\epsilon_{1}$ and $\epsilon_{2}$ along the $x$ axis in order to analyze their coupled dipole oscillations; see Fig. 1 . Since $\bar{G}_{i j}^{\mathrm{B}}$ already contains a Fourier transform with respect to $z$ momentum, see Eq. (14), the bosonic Green's functions entering Eq. (22) now simply add up to the density-density correlation function of the BEC evaluated at the $3 \mathrm{D}$ real-space distance $r=\left|\mathbf{r}_{1}-\mathbf{r}_{2}+d \hat{\mathbf{z}}\right|$. Using this, we finally obtain

$$
\begin{aligned}
\Omega_{\mathrm{mi}}\left(\epsilon_{1}-\epsilon_{2}\right)= & -\frac{m_{B} n_{B}}{\pi} \int d^{2} r_{1} d^{2} r_{2} \frac{e^{-\sqrt{2} r / \xi_{B}}}{r} \\
& \times \bar{\Omega}_{1}\left(\mathbf{r}_{1}-\epsilon_{1} \hat{\mathbf{x}}\right) \bar{\Omega}_{2}\left(\mathbf{r}_{2}-\epsilon_{2} \hat{\mathbf{x}}\right) .
\end{aligned}
$$

Equation (23) can be understood as follows. Consider two area elements of the $2 \mathrm{D}$ gases, one located at $\mathbf{r}_{1}-\epsilon_{1} \hat{\mathbf{x}}$ in layer 1 and the other at $\mathbf{r}_{2}-\epsilon_{2} \hat{\mathbf{x}}$ in layer 2 . The contribution to $\Omega_{\mathrm{mi}}$ from these two elements can be approximated by the expression in Eq. (17) in which the relative distance is taken to be $r$ instead of $d$. Equation (23) then sums up all such contributions in the two clouds.

For weak interaction, we see from Eq. (18) that $\bar{\Omega}_{j}\left(\mathbf{r}_{j}-\right.$ $\left.\epsilon_{j} \hat{\mathbf{x}}\right)=g n_{j}\left(\mathbf{r}_{j}-\epsilon_{j} \hat{\mathbf{x}}\right)$, where $n_{j}\left(\mathbf{r}_{j}-\epsilon_{j} \hat{\mathbf{x}}\right)$ denotes the equilibrium fermion density in layer $j$ rigidly displaced the distance $\epsilon_{j}$ along the $x$ axis. Equation (23) then simplifies to

$$
\begin{aligned}
\Omega_{\mathrm{mi}}\left(\epsilon_{1}-\epsilon_{2}\right)= & -g^{2} \frac{m_{B} n_{B}}{\pi} \int d^{2} r_{1} d^{2} r_{2} \frac{e^{-\sqrt{2} r / \xi_{B}}}{r} \\
& \times n_{1}\left(\mathbf{r}_{1}-\epsilon_{1} \hat{\mathbf{x}}\right) n_{2}\left(\mathbf{r}_{2}-\epsilon_{2} \hat{\mathbf{x}}\right),
\end{aligned}
$$

which is the usual Hartree approximation for the interaction energy between the two planes mediated by a Yukawa interaction.

\section{COUPLED DIPOLE OSCILLATIONS}

Consider now the situation where the two clouds perform dipole oscillations around their equilibrium positions, see Fig. 1. For small displacements $\epsilon_{1}$ and $\epsilon_{2}$, the COM velocities and the beating frequencies are small compared to the speed of sound in the 3D gas and the trapping frequencies, respectively, yielding rigid and undamped oscillations of the 2D clouds [24]. The COM dynamics is then determined by the energy increase $\delta E$ associated with the displacements of the clouds. For rigid displacements, we have $\delta E=\Omega_{\mathrm{mi}}\left(\epsilon_{1}-\epsilon_{2}\right)-\Omega_{\mathrm{mi}}(0)+$ $\left[\mu_{A}\left(\epsilon_{1}\right)+\mu_{A}\left(\epsilon_{2}\right)-2 \mu_{A}\right] N_{A}$, which gives

$$
\delta E\left(\epsilon_{1}, \epsilon_{2}\right)=\frac{1}{2} N_{A} m_{A} \omega_{\perp}^{2}\left(\epsilon_{1}^{2}+\epsilon_{2}^{2}\right)+\Omega_{\mathrm{mi}}\left(\epsilon_{1}-\epsilon_{2}\right)-\Omega_{\mathrm{mi}}(0),
$$

where $N_{A}$ is the number of fermions in each layer. Taylor expanding $\Omega_{\mathrm{mi}}\left(\epsilon_{1}-\epsilon_{2}\right)$ to second order in $\epsilon_{1}-\epsilon_{2}$, we readily see that the motion of the two clouds separates into an in-phase oscillation with frequency $\omega_{\perp}$ and an out-of-phase oscillation with frequency

$$
\omega_{r}=\omega_{\perp} \sqrt{1+2 I / N_{A} m_{A} \omega_{\perp}^{2}},
$$

where

$$
I=\left.\frac{\partial^{2}}{\partial \epsilon_{1}^{2}} \Omega_{\mathrm{mi}}\left(\epsilon_{1}-\epsilon_{2}\right)\right|_{\epsilon_{1}-\epsilon_{2}=0} .
$$

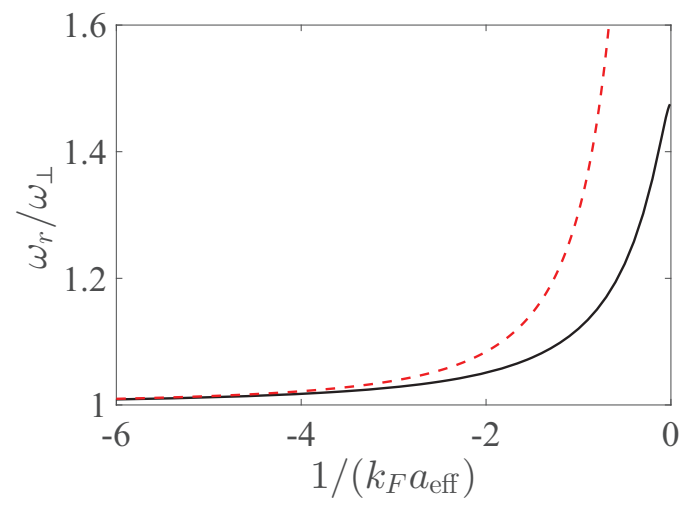

FIG. 4. The frequency ratio $\omega_{r} / \omega_{\perp}$ of the out-of-phase dipole oscillation as a function of $1 /\left(k_{F} a_{\text {eff }}\right)$. The solid line is the full strongcoupling result whereas the dashed line is determined by the secondorder perturbation theory.

The microscopic expression for $\omega_{r}$ for arbitrary strength of the 2D-3D interaction in terms of Eqs. (18), (23), (26), and (27) is the main result of this paper and it explicitly shows how the mediated interaction can be probed by measuring the frequency of the out-of-phase dipole oscillations of the two clouds.

As an example, we now calculate the frequency $\omega_{r}$ for a realistic cold-atom system consisting of $N_{A}=1000{ }^{40} \mathrm{~K}$ atoms trapped in each plane, immersed in a 3D BEC of ${ }^{6} \mathrm{Li}$ dimers. The transverse trapping frequency for the ${ }^{40} \mathrm{~K}$ clouds is $\omega_{\perp}=2 \pi \times 380 \mathrm{~Hz}$, the density of the BEC is $n_{B}=10^{18} \mathrm{~m}^{-3}$, and the coherence length is $\xi_{B}=2.7 \mu \mathrm{m}$. We furthermore assume that the temperature is zero. In Fig. 4, we show the frequency $\omega_{r} / \omega_{\perp}$ as a function of the 2D-3D interaction strength $1 / k_{F} a_{\text {eff }}$ at a fixed interlayer distance $d=0.4 \mu \mathrm{m}$. The frequency increases monotonically as $a_{\text {eff }}$ increases. For weak interaction, it agrees with the second-order result (dashed line). For stronger interaction, the full frequency and momentum dependence of the 2D-3D scattering is important, and the perturbative result deviates significantly from the full strong-coupling theory. In particular, whereas the perturbative result diverges for $1 / k_{F} a_{\text {eff }} \rightarrow 0$, the strong-coupling theory predicts a finite frequency saturating at $\omega_{r} \simeq 1.48 \omega_{\perp}$. Importantly, the frequency shift becomes significant for $-2 \lesssim 1 / k_{F} a_{\text {eff }} \leqslant 0$, which includes a region sufficiently far from unitarity so that the predicted three-body loss is small [25]. This demonstrates the usefulness of our proposal to detect mediated interactions. Note that this result can only be obtained using a strong-coupling theory, since the perturbative result is only accurate for weak interactions where the frequency shift is minute.

In Fig. 5, we plot $\omega_{r} / \omega_{\perp}$ as a function of the ratio of the interparticle distances $n_{B}^{1 / 3} / n_{F}^{1 / 2}$ (keeping $n_{F}$ fixed) with $1 / k_{F} a_{\text {eff }}=-0.1$ and all other physical parameters the same as for Fig. 4(a). The density of the BEC enters the mediated interaction in two ways, which is most clearly seen in the weak-coupling limit given by Eq. (3): First, the strength of the interaction is proportional to $n_{B}$; second, the range of the interaction is determined by the BEC coherence length $\xi_{B} \propto 1 / \sqrt{n_{B}}$. Thus, increasing the density increases the strength but reduces the range of the mediated interaction, 


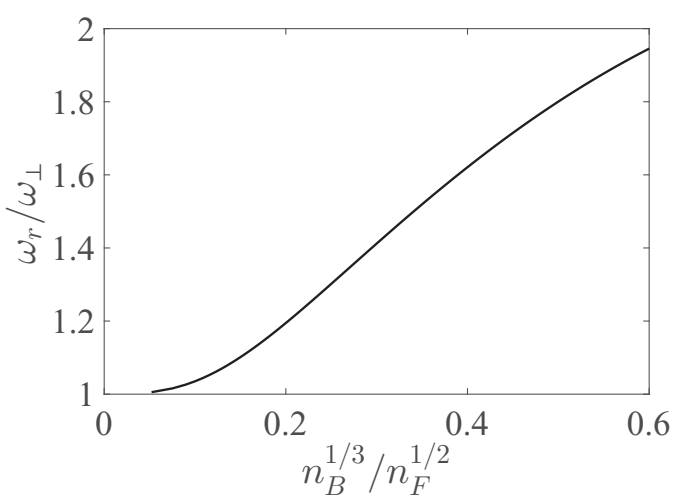

FIG. 5. The frequency ratio $\omega_{r} / \omega_{\perp}$ as a function of the ratio of the interparticle distances $n_{B}^{1 / 3} / n_{F}^{1 / 2}$ (keeping $n_{F}$ fixed) for $1 /\left(k_{F} a_{\mathrm{eff}}\right)=$ -0.1 and all other parameters as in Fig. 3. Here $n_{F}$ is the fermion density at the center of the cloud.

and it is not a priori obvious what the net effect on the frequency shift will be. From Fig. 5, we see that for the chosen parameters, $\omega_{r}$ in fact increases monotonically with increasing BEC density. Finally we point out that we have restricted all calculations to negative values of the 2D-3D scattering length. Indeed for $1 / k_{F} a_{\text {eff }}>0$, a $2 \mathrm{D}$ fermion can form a bound-dimer state with a $3 \mathrm{D}$ boson. The frequency shift in this region therefore depends on whether the system forms these dimers, or whether it is on the so-called repulsive branch where the effective 2D-3D interaction is repulsive. This more complicated situation will be investigated in future studies.

\section{CONCLUDING REMARKS}

In this paper, we demonstrated that a mixed-dimensional setup consisting of two layers of identical fermions immersed in a $3 \mathrm{D}$ background gas is a powerful probe to investigate mediated interactions systematically. The mediated interaction between the two layers modifies the out-of-phase dipole oscillation frequency of the $2 \mathrm{D}$ clouds, and we calculate this shift using a strong-coupling theory taking into account the low-energy scattering between the $2 \mathrm{D}$ and $3 \mathrm{D}$ particles. Using this theory, we showed that for strong 2D-3D coupling, the resulting frequency shift is clearly measurable.

Finally we note that the advantages of our proposal are twofold. First, if the 2D trapping is realized using optical potentials, the distance between planes is a few hundred nanometers, which is much larger than the range of interatomic interactions. Any observed coupling between the two planes is therefore solely due to a mediated interaction via the $3 \mathrm{D}$ gas. Second, the shift of the center-of-mass oscillation frequency is a very precise spectroscopic tool that can be used as a probe of weak interactions, as demonstrated recently in [24,26].

\section{ACKNOWLEDGMENTS}

F.C. and D.S. acknowledge support from Région Ile de France (DIM IFRAF/NanoK), ANR (Grant No. SpiFBox), and the European Union (ERC Grant No. ThermoDynaMix). G.M.B. and Z.W. wishes to acknowledge the support of the Villum Foundation via Grant No. VKR023163.

D.S. and Z.W. contributed equally to this work.
[1] S. Weinberg, The Quantum Theory of Fields, Vol. 1 (Cambridge University Press, New York, 1995).

[2] J. R. Schrieffer, Theory of Superconductivity, Advanced Book Program Series (Perseus Books, Reading, Massachusetts, 1983).

[3] D. J. Scalapino, The case for $d_{x^{2}-y^{2}}$ pairing in the cuprate superconductors, Phys. Rep. 250, 329 (1995).

[4] K. A. Milton, The Casimir Effect: Physical Manifestations of Zero-Point Energy (World Scientific, Singapore, 2001).

[5] B. B. Machta, S. L. Veatch, and J. P. Sethna, Critical Casimir Forces in Cellular Membranes, Phys. Rev. Lett. 109, 138101 (2012).

[6] A. Bulgac, M. M. N. Forbes, and A. Schwenk, Induced $P$-Wave Superfluidity in Asymmetric Fermi Gases, Phys. Rev. Lett. 97, 020402 (2006).

[7] C. Lobo, A. Recati, S. Giorgini, and S. Stringari, Normal State of a Polarized Fermi Gas at Unitarity, Phys. Rev. Lett. 97, 200403 (2006).

[8] C. Mora and F. Chevy, Normal Phase of an Imbalanced Fermi Gas, Phys. Rev. Lett. 104, 230402 (2010).

[9] Z. Yu, S. Zöllner, and C. J. Pethick, Comment on "Normal Phase of an Imbalanced Fermi Gas”, Phys. Rev. Lett. 105, 188901 (2010).

[10] Z. Wu and G. M. Bruun, Topological Superfluid in a Fermi-Bose Mixture with a High Critical Temperature, Phys. Rev. Lett. 117, 245302 (2016).

[11] J. Melkær Midtgaard, Z. Wu, and G. M. Bruun, Topological superfluidity of lattice fermions inside a Bose-Einstein condensate, Phys. Rev. A 94, 063631 (2016).
[12] M. A. Caracanhas, F. Schreck, and C. Morais Smith, Fermi-Bose mixture in mixed dimensions, arXiv:1701.04702.

[13] M. Schecter and A. Kamenev, Phonon-Mediated Casimir Interaction between Mobile Impurities in One-Dimensional Quantum Liquids, Phys. Rev. Lett. 112, 155301 (2014).

[14] Y. Nishida, Phases of a bilayer Fermi gas, Phys. Rev. A 82, 011605 (2010).

[15] A. G. Rojo, Electron-drag effects in coupled electron systems, J. Phys.: Condens. Matter 11, R31 (1999).

[16] N. Matveeva, A. Recati, and S. Stringari, Dipolar drag in bilayer harmonically trapped gases, Eur. Phys. J. D 65, 219 (2011).

[17] Y. Nishida and S. Tan, Universal Fermi Gases in Mixed Dimensions, Phys. Rev. Lett. 101, 170401 (2008).

[18] Y. Nishida, Induced $p$-wave superfluidity in two dimensions: Brane world in cold atoms and nonrelativistic defect CFTs, Ann. Phys. 324, 897 (2009).

[19] M. A. Ruderman and C. Kittel, Indirect exchange coupling of nuclear magnetic moments by conduction electrons, Phys. Rev. 96, 99 (1954).

[20] T. Kasuya, A theory of metallic ferro- and antiferromagnetism on Zener's model, Prog. Theor. Phys. 16, 45 (1956).

[21] K. Yosida, Magnetic properties of Cu-Mn alloys, Phys. Rev. 106, 893 (1957).

[22] H. Yukawa, On the interaction of elementary particles, Proc. Phys. Math. Soc. Jpn. 17, 48 (1935). 
[23] D. S. Petrov, C. Salomon, and G. V. Shlyapnikov, Weakly Bound Dimers of Fermionic Atoms, Phys. Rev. Lett. 93, 090404 (2004).

[24] I. Ferrier-Barbut, M. Delehaye, S. Laurent, A. T. Grier, M. Pierce, B. S. Rem, F. Chevy, and C. Salomon, A mixture of Bose and Fermi superfluids, Science 345, 1035 (2014).
[25] Y. Nishida and S. Tan, Liberating Efimov physics from three dimensions, Few-Body Syst. 51, 191 (2011).

[26] R. Roy, A. Green, R. Bowler, and S. Gupta, Two-Element Mixture of Bose and Fermi Superfluids, Phys. Rev. Lett. 118, 055301 (2017). 